\title{
Multivariate Analysis of walker-assisted ambulation
}

\author{
M. Martins, C. P. Santos \\ Industrial Electronics Department \\ University of Minho \\ Guimarães, Portugal \\ mariam,cristina@dei.uminho.pt
}

\author{
L. Costa \\ Production and Systems Department \\ University of Minho \\ Guimarães, Portugal \\ lac@dep.uminho.pt
}

\author{
A. Frizera-Neto \\ Electronics Department \\ UFES \\ Vitória, Brazil \\ anselmo@ele.ufes.br
}

\begin{abstract}
In an aging society it is extremely important to develop devices which can support and aid the elderly in their daily life. Walkers play an important role, due to the large number of potential users, its simplicity and their ambulatory potential. However, there are no clinical evidences that prove the efficacy of such devices, mainly rollators that present forearm supports. In this context, the authors aim to propose a protocol for an innovative gait analysis that addresses some benefits and limitations of these devices on the rehabilitation process, by addressing a multivariate analysis of spatiotemporal and kinematic gait parameters assessed during normal and assisted ambulation with a walker with forearm supports. For the 3Dreconstruction of the body segments it was used a movement analysis system. Results showed that the effects of assisted gait can be explained through support, energy consumption, posture and balance characteristics. These results are very satisfactory since aspects regarding these characteristics enhance the rehabilitation potential of the use of walkers with forearm supports. These results will be used to advance towards an active robotic walker that will provide for safety and natural manoeuvrability and offer a certain degree of intelligence in assistance and decision-making.
\end{abstract}

Keywords - walker-assistive gait, Rehabilitation, Multivariate Analysis.

\section{INTRODUCTION}

Assisting patients that suffer from mobility disabilities has become one of the most important concerns on the field of Rehabilitation Robotics. Considering users with partial mobility, walker devices appeared showing a great potential in rehabilitation and functional compensation of moderate to severe disabilities. This potential arises due to the use of the user's residual capabilities, maintaining and enhancing motor functions by means of daily training with the device.

However, studies [1,2] have shown that $30-50 \%$ of people abandon their walkers soon after receiving them. These results raise questions about the effectiveness, selection, proper training, design and potential problems that may be related to this. Additionally, most patients have never been instructed on the proper use of these devices and often have devices that are inappropriate, damaged, or are of the incorrect height. In [1], Bradley presents a list of pros and cons of the walker devices, highlighting that selection of a suitable device depends on the patient's strength, endurance, balance, cognitive function, and environmental demands. However, this is based on visual observation, which is not accurate in terms of the walker's effects on the user biomechanics, and consequent rehabilitation process.

There is not great diversity of studies in the literature, or metrics that show with great evidence, in a quantitative way, the limitations and benefits that walkers can bring to their target users. In addition, the found studies focus on the standard frames and rollators with conventional handlebars.

In this study, the authors focus on walkers with forearm supports. Such devices provide better partial body weight support [4], which is essential for the patient's rehabilitation. This helps to reduce load on the affected lower limbs, alleviating them and allowing a better development of gait patterns.

In this context, this paper proposes a protocol for an innovative gait analysis that addresses some benefits and limitations of these devices on the rehabilitation process. It applies a Principal-Component Analysis (PCA) as a multivariate analysis tool suitable for gait data evaluation [6]. This method allows a convenient summary of the many gait variables called principal components. So, this study will show how PCA can provide insight into usefulness of gait data with and without walker, i.e. have an insight into similarities and differences in gait performances arising from using/nonusing the walker.

Most important contribution of this study is presenting first steps for the classification of temporal and high dimensional gait data with the use of walker with forearm supports, which does not exist in the literature.

This paper is organized as follows. Section II presents the methods, which include a brief description of the participants, experimental setup and statistical analysis. Section III presents the acquired results with the application of PCA. Section IV discusses the results. Finally, conclusions are discussed in Section IV.

\section{METHODS}

\section{A. Participants}

In total 13 healthy young (age range 23-27 years) participants, 7 males and 6 females, with any history of gait dysfunction or injuries in the lower limbs were recruited to participate in this study (Table I). 
TABLE I. SUBJECTS CHARACTERISTICS

\begin{tabular}{|c|c|c|c|}
\hline & Age & Weight $(\mathrm{Kg})$ & Height $(\mathrm{m})$ \\
\hline Mean \pm SD & $26.84 \pm 4.55$ & $68.76 \pm 10.74$ & $1.76 \pm 0.08$ \\
\hline
\end{tabular}

\section{B. Experimental Procedure}

For this study, it was defined a specific protocol of experimentation, since there are no accepted guidelines in the literature for this type of walker. The first parameter of interest is the velocity of gait. It has been established that the user must walk with an arbitrary and preferred speed when using the device. This is due to the fact that it is desirable to know which is the natural gait speed developed when using the walker. In addition, the authors did not intend to force specific patterns by fixing a certain speed.

Another important parameter is the height of the forearmsupport. It has been established as a starting point that the height of forearms should be equal to the height measured between the elbow of the user and the ground. Thus, the subjects should assume a standing upright position with their forearms placed in the supports of the walker. The lower extremities have to be in a vertical position with the knee joints extended, and the upper extremities flexed with an elbow flexion angle of 90 degrees when he is supported by the device at rest $[4,8]$.

Once these criteria were established, different experiments were done which will be analysed and discussed next.

Experiments were performed using the VICON 612 motion analysis system (http://www.vicon.com/) connected to six video cameras at a frequency of $200 \mathrm{~Hz}$. VICON can create a $3 \mathrm{D}$ motion model by using the spatial positions of particular markers placed on the user.

The subjects were fitted with fifteen reflecting spherical markers according to the marker set-up described by Vaughan et al [9]. All subjects were barefoot and asked to perform two sessions 4 times each: they had to walk forward in a straight line (1) without and (2) with the walker. Thus, two groups of study were considered: unassisted and assisted gait users.

\section{Data Processing}

Custom Matlab Software (Mathworks, Natick, MA, USA) was used for all data processing, calculations and analysis.

The variables selected for statistical analysis can be grouped into 2 categories: (1) spatiotemporal and (2) joint kinematics. Considering that the subjects present no motor dysfunctions, symmetrical gait was assumed [10]. The definitions are shown in Table II.

The variables used were those commonly reported in gait analysis. Joint angles were calculated using a 3-D Euler rotation sequence (Z-Y-X). Median over 4 trials were calculated for each variable of subject, and formed the basis for all subsequent analyses.
TABLE II. DEFINITION OF GAIT ANALYSIS VARIABLES

\begin{tabular}{|c|c|}
\hline Name & Variable \\
\hline Step width (m) & Width \\
\hline Step length (m) & Step 1 \\
\hline Cadence (m/min) & CAD \\
\hline Stance phase(\%) & stance \\
\hline Swing phase (\%) & swing \\
\hline Double support (\%) & DS \\
\hline Average Speed (m/s) & Speed \\
\hline Step time (s) & Step_t \\
\hline Ankle plantarflexion maximum (degrees) & APF \\
\hline Ankle dorsiflexion maximum (degrees) & ADF \\
\hline Ankle range of motion during gait cycle (degrees) & ATz \\
\hline Maximum flexion of the knee (degrees) & KF \\
\hline Maximum extension of the knee (degrees) & $\mathrm{KE}$ \\
\hline Knee range of motion during gait cycle (degrees) & KT \\
\hline Maximum flexion of hip (degrees) & $\mathrm{HF}$ \\
\hline Maximum extension of hip (degrees) & $\mathrm{HE}$ \\
\hline Hip range of motion during gait cycle in $\mathrm{z}$ (degrees) & HT \\
\hline Maximum abduction of the hip (degrees) & $\mathrm{Hab}$ \\
\hline Maximum adduction of the hip (degrees) & Had \\
\hline Hip range of motion during gait cycle in $x$ (degrees) & HTx \\
\hline $\begin{array}{c}\text { Foot maximum progression deviation (interior rotation) } \\
\text { (degrees) }\end{array}$ & $\mathrm{AI}$ \\
\hline $\begin{array}{c}\text { Foot maximum progression deviation (exterior rotation) } \\
\text { (degrees) }\end{array}$ & $\mathrm{AE}$ \\
\hline $\begin{array}{c}\text { Foot range of progression deviation during gait cycle } \\
\text { (degrees) }\end{array}$ & ATx \\
\hline Range of motion of sacrum (height) (m) & $\mathrm{T} 10 \mathrm{z}$ \\
\hline Lateral flexion (right) of the trunk (degrees) & SR \\
\hline Lateral flexion (left) of the trunk (degrees) & $\mathrm{SL}$ \\
\hline Pelvic lateral range of motion (degrees) & ROMlat \\
\hline Pelvic maximum flexion (degrees) & SF \\
\hline Pelvic maximum extension (degrees) & SE \\
\hline Range of motion of Ext/Flex of the trunk (degrees) & ROMFlexExt \\
\hline total & 30 \\
\hline
\end{tabular}

\section{Statistical Analysis}

In this application, PCA was applied to an $n \times p$ matrix, where $n$ is the participants and $p$ is the variables, which are 30 with previous normalization. Due to the existence of two groups of study (assisted and non-assisted users) with 13 users each one, this resulted in a $26 \times 30$ matrix. Further, it was constructed a normalized covariance matrix.

The Principal Components (PCs) were constructed successively as follows. First PC (PC1) is the linear combination of variables for which the variation between subjects was the greatest (\%), that is, it goes as far as possible to explain the differences among the subjects. The second PC (PC2) worked in the same fashion, using the variability that was left after the first PC had been removed. This process is repeated for all the other PC's. A scree graph was used to indicate the proportion of variance explained by each PC (Fig.1)

The number of PCs to select is performed by keeping the first few principal components which retain the most variance of the data, as follows. The first criterion is to select PC's with eigenvalues greater than 1 . Second criterion is to determine how many PC's are necessary to retain in the model $60-70 \%$ trace, meaning that only the PCs required to explain $60-70 \%$ of the data variance were kept and the remaining PCs were discarded, effectively achieving data reduction. 


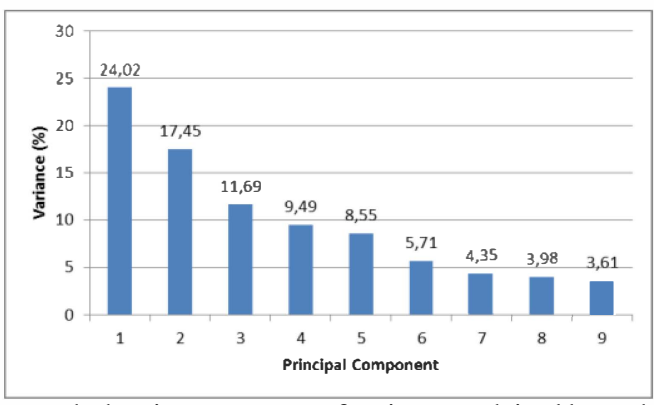

Fig. 1 Scree graph showing percentage of variance explained by each of the first 9 principal components.

By choosing the first four PCs, $63 \%$ of the variation of gait variables is considered. Thus, the authors assume that four PC's are enough to represent our problem, since they represent $63 \%$ of the variance of gait variables.

The obtained results are discussed in the following section.

\section{RESULTS}

Table III presents the variables with the higher loadings for the four most important components.

PC1 is positively high correlated with six variables and negatively high correlated with five variables. These variables indicate that PC1 is related to the kind of walking support the walker can provide to the user.

PC2 is positively high correlated with six variables and negatively high correlated with four variables. These variables indicate that PC2 is related to the consume of energy and the posture that the user acquires when walks with and without the walker.

PC3 is positively high correlated with six variables and negatively high correlated with four variables. These variables indicate that PC3 is related to balance, thus giving the opportunity to evaluate the kind of help that the device provides to the user. For example, it can be evaluated if the user has posture problems, or good biomechanical stability.

Finally, PC4 seems to be redundant since it contains most of the variables that were retained in $\mathrm{PC} 2$, and does not seem to generalize any specific feature to this study. Therefore, PC4 it will not be considered in this study.

\section{DISCUSSION}

This section address a detailed analysis of each of the three principal components previously discussed in section II, and attempts to identify the differences between assisted and nonassisted gait.

\section{A. PC1-Support}

According to the obtained results, it was verified that $\mathrm{PC} 1$ is related to the effects that the walking support provided by the walker can have in the gait variables.

When comparing unassisted and assisted gait, the first one presents greater step length (step_l), which leads to a greater swing duration (swing). The fact that the user presents a larger step length augments the total knee and ankle excursion ( $K T$ and $A T z$ ), mainly the knee flexion. The extra support, provided by the walker-assisted ambulation, causes the increase of stance duration (stance), with a more secure and careful step (this variable is the opposite of the swing). Since the user has more stability, the range of motion of the knee is lower and flexes less the ankle. This is consistent with the results obtained in [8].

TABLE III. VARIABLES CORRELATING WITH EACH OF THE FOUR PC'S.

\begin{tabular}{|c|c|c|c|c|c|c|c|}
\hline PC1 & Loading & PC2 & Loading & PC3 & Loading & PC4 & Loading \\
\hline KF & 0,30339 & speed & 0,355001 & HF & 0,389844 & SF & 0,370925 \\
\hline swing & 0,283252 & CAD & 0,295305 & APF & 0,380103 & SE & 0,319235 \\
\hline KT & 0,282337 & Had & 0,239545 & ADF & 0,260581 & KE & 0,30103 \\
\hline step_I & 0,23416 & T10z & 0,236481 & step_I & 0,239329 & ADF & 0,288401 \\
\hline Atz & 0,20658 & SF & 0,235043 & HT & 0,212714 & width & 0,269998 \\
\hline SR & $-0,25277$ & ROMFlexExt & 0,220411 & HE & 0,203796 & AE & 0,257436 \\
\hline ROM lat & $-0,26516$ & DS & $-0,27336$ & width & $-0,20307$ & Atz & 0,214212 \\
\hline stance & $-0,27335$ & HTx & $-0,28844$ & Al & $-0,22929$ & HT & $-0,20981$ \\
\hline SL & $-0,2933$ & Step_t & $-0,31393$ & DS & $-0,27611$ & T10z & $-0,24033$ \\
\hline- & - & Hab & $-0,1741$ & AE & $-0,28336$ & - & - \\
\hline
\end{tabular}

Other observation is the increase of the lateral movement of the pelvis (ROMlat). This means that the user is decreasing the load of the body on the lower limbs, thus directing this load to the trunk/upper limbs. This can be an advantage in case the user has strong arms, weak legs and if he is an occasional walker user, this may be adequate. However, for a person with painful hand and wrist problems, daily use of a simple walker over several years to decades may be deleterious. Consequently, patients may opt to sit in a wheelchair, which leads to further physical deconditioning and joint contractures and may preclude future attempts to regain the ability to walk, decreasing the person's access to environment and work [10]. The upper limb has not been well studied as a weight-bearing limb. In [11], Bachschmidt et al concluded that walker assisted gait with handgrip can be a demanding task for the elbow extensors, wrist flexors and shoulder flexors and adductors. However, there's still no specific study of the effect that the forearm supports have on the shoulder, elbow and wrist joints.

\section{B. PC2 - Energy Consumption and Posture}

PC2 is related to the effects that the use of the walker can have in the posture and speed of the user.

The use of the walker decreased the speed (speed) and the cadence $(C A D)$, as the step is longer in time $\left(\right.$ step $\left._{-} t\right)$ and shorter in distance.

Concerning posture during the walker gait in this study, the forearms were supported by the platforms, restricting the movement of the upper limbs to present a constant position of the shoulders. Therefore, the vertical movement of the trunk $(\mathrm{TlOz})$ is reduced to small movements, preventing great 
displacements of the body's CoG. This effect was previously observed in [12] and measured experimentally in [13].

Also, the unassisted gait presents greater flexion/extension of the pelvis (ROMFlexExt, SF) in comparison with the assisted gait, i.e. less stable posture meaning a greater range of motion in the sagittal plane of the pelvis. So, it can be verified that the walker helped to improve posture, since flexion and extension of the pelvis decrease with its use.

Hip adduction (Had) increases with the use of the walker (is a negative angle), as well as hip abduction (Hab).

\section{PC3 - Balance}

PC3 can be associated to the effects that the use of the walker can have in the user's balance.

Results show that the use of the walker improved balance by decreasing step width (width) and increasing the double support $(D S)$. It is also noticed that some of the walker users present a greater hip flexion $(H F)$ and extension $(H E)$. This can be due to the elbow joint flexion that should be $90^{\circ}$ in order to do not influence the hip joint. This fact could be associated with low back pain [12]. However, a study made by Ishikura [12], found that there is less muscle activity on the lumbar erector spinae than during normal gait, and the chance of fatigue occurring to be minimal. Thus, the possibility of low back pain occurring is considered to be scarce.

Reinforcing the idea of greater balance, it is observed that since the user is supported, he should decrease his load on the legs, decreasing the dorsiflexion $(A D F)$ and plantarflexion $(A P F)$ of the ankle. Results confirm these assumptions. Foot progression deviation ( $A E$ and $A I)$ also seems to decrease with the use of the walker, which means that he is well-supported and with good posture.

\section{CONCLUSIONS}

This study proposed a protocol for an innovative gait analysis to address the benefits and limitations of assisted gait using a walker with forearm supports. It intends to present the first steps for the classification of the acquired gait data with this device, which does not exist in the literature.

It have been identified three PCs that explain about $63 \%$ of the variation in gait measures of persons without any gait dysfunction, when they are walking with and without a walker.

The first PC is related to support, the second is related to energy consumption and posture and the last one with balance. These results are very satisfactory since aspects regarding posture, balance and support enhance the rehabilitation potential of the use of walkers with forearm supports.

Important clinical observations were also made: it was observed that it is essential that the supporting platforms are sufficiently high to impose an upright posture to the patient. This is to avoid the increase in hip and pelvis flexion, which can lead to back pain [12]; and, there was an increase in the lateral pelvic motion of the users when performing assisted gait. This observation should be considered by physicians when prescribing this device to patients with lower limbs limitations.

Thus, the proposed work allows data reduction in gait analysis, by investigating, in an objective and statistical way, the relationships between the vast quantities of variables and numerical information.

However, further research is necessary, to fully exploit this type of analysis to clinical validation, with elderly users, poststroke, and other dysfunction gait patients. This future research should also analyze the impact that weight-bearing strategies based of forearm supporting platforms have on patients with injuries on the upper limbs articulations, as well as, the effects of long-time usage.

\section{ACKNOWLEDGMENT}

This work is financiered by FEDER Funds and through Programa Operacional Fatores de Competitividade COMPETE and by National Funds through FCT - Fundação para a Ciência e Tecnologia under the Project: FCOMP-010124-FEDER-022674. Work supported by Portuguese Science Foundation (grant SFRH/BD/76097/2011).

\section{REFERENCES}

[1] Bradley, S., Hernandez, C. 2011. "Geriatric Assistive Devices". American Family Physician, vol 84, nº4.

[2] Bateni, H., Maki, B.E. 2005. "Assistive devices for balance and mobility: benefits, demands and adverse consequences. Arch. Phys. Med. Rehabil. 8G, 134-145.

[3] Van Hook, F.W, Demonbreun, D., Weiss, B.D. 2003. "Ambulatory devices for chronic gait disorders in the elderly. Am Fam Physician. 67(8):1717-1724.

[4] Youdas, J., Kotajarvi, B., Padgett, D., Kaufman, K. 2005 "Partial weight-bearing gait using conventional assistive devices". Arch Phys Med Rehabil, vol. 86, 394-398.

[5] Olney, S., Griffin, M., McBride, I. 1998 "Multivariate Examination of Data from Gait Analysis of Persons with Stroke". Journal of the American Physical Therapy Association. 78:814-828.

[6] Jolliffe, I. "Principal Component Analysis, 2nd Edition". Springer.

[7] Whittle, M.“Gait Analysis - An Introduction”. 4th Editio, Elsevier

[8] Alkjaer, T., Larsen, P., Pedersen, G., Nielsen, L., Simonsen, E. 2006 "Biomechanical analysis of rollator walking". Biomedical Engineering Online 5:2, doi:10.1186/1475-925X-5-2

[9] Vaughan C, Davis B, Connor J. Dynamics of human gait. Kiboho publishers, 2nd ed.

[10] Chiou-Tan, F., Magee, K., Krouskop, T. 1999. "Comparison of upper limb muscle activity in four walking canes: a preliminary study". Journal Rehabilitation Res. Dev. 36:94.9.

[11] Bachschmidt, R., Harris, G., Simoneau, G. 2001. "Walker-assisted gait in rehabilitation: a study of biomechanics and instrumentation. IEEE Trans. Neural Syst. Rehal. Eng 9:96-105.

[12] Ishikura, T.2001. "Biomechanical Analysis of weight bearing force and muscle activation levels in the lower extremities during gait with a walker". Acta Med. Okayama,vol.55, n², pp. 73-82.

[13] Frizera, A ; Abellanas, A. ; Ceres, R. ; Pons, J. L. "Analysis of ground reaction forces in walker assisted gait" In: 10th European Conference for the Advancement of Assistive Technology in Europe , Florence, 2009, v. 25. p. $450-454$. 